REGARDS

SUR L'ECONOMIE ALLEMANDE

BULLETIN ECONOMIQUE DU CRAC

\section{Regards sur l'économie allemande}

Bulletin économique du CIRAC

$65 \mid 2004$

Varia

\title{
Consommation : du nouveau du côté des chômeurs
}

\section{Natacha Dagneaud}

\section{OpenEdition}

Journals

Édition électronique

URL : http://journals.openedition.org/rea/3949

DOI : 10.4000/rea.3949

ISBN : 978-2-8218-0826-3

ISSN : 1965-0787

Éditeur

CIRAC

Édition imprimée

Date de publication : 1 mars 2004

Pagination : 35-36

ISSN : 1156-8992

Référence électronique

Natacha Dagneaud, "Consommation : du nouveau du côté des chômeurs », Regards sur l'économie allemande [En ligne], 65 I mars 2004, mis en ligne le 29 octobre 2009, consulté le 15 septembre 2020. URL : http://journals.openedition.org/rea/3949

Ce document a été généré automatiquement le 15 septembre 2020

(C) CIRAC 


\title{
Consommation : du nouveau du côté des chômeurs
}

\author{
Natacha Dagneaud
}

1 L'Allemagne compte près de 4,4 millions de sans emploi. Le montant global des revenus de substitution dont ils disposent s'élève à plus de 30 milliards $€$. Quelle réalité recouvrent ces données macroéconomiques? Quels types de consommateurs sont ces personnes hier encore employées et aujourd'hui au chômage ? Une étude exploratoire sur le comportement d'achat des chômeurs parue mi-janvier, réalisée par l'institut d'études de marché Séissmo (Mannheim, www.seissmo.com), vient mettre un terme à une série d'idées reçues largement ancrées dans une partie du monde de l'industrie et des biens de consommation. Les hommes et les femmes de 26 à 55 ans, inscrits au chômage depuis plus de 4 mois et bénéficiaires des divers types d'allocations et aides, méritent d'être considérés comme une cible marketing à part entière. L'étude Séissmograph 2003 révèle toute la diversité socioculturelle qu'elle contient et tout le potentiel économique qu'elle renferme.

\section{Une grande variété de profils}

2 «Les» chômeurs n'existent pas plus que «les» actifs occupés. Au contraire, ils présentent une large variété de profils :

le type actif, qui conserve l'esprit combatif et une certaine ambition;

- le type contenté, qui arrive à s'arranger de sa situation momentanée et qui, globalement, la vit sereinement ;

l'autruche, qui n'accepte pas son nouveau statut et développe des conduites de fuite devant la réalité ;

le champion du système $D$, qui tire habilement parti de ses nouvelles conditions de vie ;

le type dépressif, qui se sent remis en cause dans son identité, qui doute fortement de lui et a presque « abandonné». 
8 Mais on trouve également de nombreux points communs à tous ces types lorsque le regard se porte sur leur mode de consommation.

\section{Sans travail ne signifie pas sans exigences}

9 Les chômeurs ont des centres d'intérêts et des loisirs pour lesquels ils se montrent dépensiers. On note partout un phénomène de " high involvement » dès qu'un thème les concerne personnellement. De façon surprenante, presque aucun chômeur, malgré la baisse de revenu qui intervient, ne se «serre la ceinture » à tous les niveaux. Au lieu d'une réduction unilatérale de la consommation, on observe des arbitrages intelligents dans les dépenses. Aussi privation et opulence coexistent-elles chez une même personne. Un besoin fort de marques se fait ressentir car elles représentent des repères importants et incarnent des valeurs que l'on peut « acheter », permettant de vivre par procuration. Cela n'exclut pourtant pas une attitude critique ni même le fait de se sentir "obligé » de justifier et légitimer par toute sorte d'argumentaires rationnels (pour la santé, le tonus, pour se former...) ce type de dépense a priori déraisonnable : on note que les chômeurs n'osent pas avouer qu'il leur arrive de se faire plaisir. Cela est particulièrement vrai dans le domaine cosmétique, visiblement lié à l'intégrité physique et au respect de soi, dans lequel on observe une grande fidélité aux marques, garantes d'authenticité.

\section{Sans travail ne signifie pas sans opinion}

10 La quête d'informations a la priorité dans le combat pour la survie, les chômeurs sont par conséquent bien informés au niveau social, personnel ainsi qu'au niveau des produits. Les chômeurs sont grands consommateurs de médias et, plus généralement, recourent à toutes les sources d'information. La collecte d'informations est certes un passe-temps, mais répond bien plus encore à une nécessité, car elle nourrit le processus d'intégration. Celui qui «sait» a le pouvoir. L'appropriation d'informations est fondamentale pour la construction identitaire.

\section{Sans travail ne signifie pas sans moyens}

11 Les chômeurs disposent d'un revenu net non négligeable et qu'ils aiment dépenser. La réalité des situations au niveau des revenus est très complexe et ne supporte pas de généralisations. Et certainement pas celles qui affirment que les chômeurs ont systématiquement moins de revenus à disposition que les actifs. L'étude a pu observer une grande variété de cas qui relativise la valeur du «revenu». Chaque situation individuelle dépend de la présence d'un partenaire actif ou non, de la présence d'enfants au foyer ou non, de la hauteur des frais au quotidien (certains sont bien aidés par leurs proches, d'autres se battent pour pouvoir payer leur loyer...). Mais pour quasiment tous, dépenser de l'argent constitue un acte de plaisir, procure un sentiment de densité du vécu et donne l'illusion de vivre une vie plus intense. Ce n'est rien d'autre que le modèle de société tout entier qui perce au travers... 


\section{Sans travail ne signifie pas sans élan}

Les chômeurs manifestent un comportement de fréquentation de magasins et enseignes (parmi une liste prédéfinie pour les besoins de l'étude) similaire à celui des actifs. En effet, le fait de sortir de chez soi et de rencontrer d'autres personnes est très important. Le monde extérieur procure le sentiment de «faire partie» du tout et renvoie une image de normalité du quotidien. Les chômeurs aiment être témoins des phénomènes de masse et sentir une forte activité autour d'eux pour sentir qu'ils appartiennent à la société.

\section{Sans travail ne signifie pas sans influence}

13 Le chômage conditionne et transforme les rôles homme-femme. La phase de chômage est une période difficile à vivre, spécialement pour les hommes qui perdent leur rôle de protecteur et leur statut de " pourvoyeur d'argent de la famille ». Ils doivent apprendre à considérer d'autres aspects dans la relation au partenaire et aux autres membres de la famille. Cependant, quelques personnes arrivent à faire du chômage une phase positive et bénéfique aux finances du foyer, en recherchant les bonnes affaires ou en comparant les prix de façon pointue.

\section{Garder le contact avec la société}

Tout, dans le comportement de consommation des chômeurs allemands, indique qu'ils tiennent particulièrement à préserver le contact avec la société. Le débat sur la fusion de l'aide au chômeur (Arbeitslosenhilfe) et de l'aide sociale (Sozialhilfe) qui occupait l'espace public au moment de l'étude était perçu, par la plupart d'entre eux, comme un acte d'exclusion et de mépris. L'adoption de cette fusion, intervenue depuis dans le cadre des réformes votées peu avant Noël, devrait confirmer cette tendance.

Le chômage est de plus en plus présent dans les mentalités en Allemagne, et il est probable que des transformations profondes de la société allemande en seront la conséquence - à commencer par un travail de deuil sur la fin de l'ère de la prospérité (Wohlstandsgesellschaft). L'éventualité du chômage risque de bousculer également la planification et la répartition famille-travail-foyer. Au-delà, la raréfaction du travail risque d'amener une nouvelle définition et un nouveau statut de celui-ci. La vocation personnelle et le fait d'aller gagner de l'argent seront (ou pourront être) vus comme légitimement distincts. Le "métier» (Beruf), qui est aux Allemands ce que le diplôme est aux Français, devrait alors jouer un rôle moins important qu'aujourd'hui dans la construction de l'identité sociale. Enfin, la fonction du chef de famille, de l'homme nourricier (Ernährer), très présente encore dans la culture allemande, s'en trouvera sérieusement remise en question, accélérant la perte de repères identitaires due au processus de dilution des attributs distinctifs masculin-féminin. (ND) 
INDEX

Mots-clés : consommation, chômage, marché du travail, emploi, travail, protection sociale, couverture sociale, assurance sociale, système social, salaire, revenu 